\title{
In silico Analysis of Compounds from Stemona tuberosa as an Inhibitor for N1 Neuraminidase of H5N1 Avian Virus
}

\author{
Abhilash Manohar \\ IBM India Pvt.Ltd,Manyta Tech Park, Bangalore - India
}

\begin{abstract}
The worldwide spread of H5N1 avian influenza has raised concerns that this virus might acquire the ability to pass readily among humans and cause a pandemic.Two anti-influenza drugs currently being used to treat the infected patients are oseltamivir (Tamiflu) and zanamivir (Relenza), both of which target the neuraminidase enzyme of the virus. Reports of the emergence of drug resistance has made the development of new anti-influenza molecules a priority. Various compounds present in Stemona tuberosa a plant belonging to Stemonaceae family was tested for activity against H5N1 neuraminidase. Eight one molecules including stilbenoids, bibenzyls and various others were selected as probable compounds for lead molecules. These lead molecules were tested for toxicity tests and Lipinski rule in Pre ADMET server. Around 33 compounds cleared all these tests. Validated ligand molecules were docked against H5NI Neuraminidase active site residues using AUTODOCK 4 which showed better results in comparison with zanamivir and oseltamivir, anti- influenza drugs.
\end{abstract}

Key words: H5N1 Avian virus, Neuraminidase inhibiotors, Stemona tuberosa, Docking analysis

\section{INTRODUCTION}

Avian influenza or "bird flu" is a contagious disease that is hosted by the birds, which infects several species of mammals. H5N1 has evolved into a flu virus strain that infects more species than any previously known flu virus strain, is deadlier and more pathogenic. The worldwide spread of $\mathrm{H} 5 \mathrm{~N} 1$ avian influenza has raised concerns that this virus might acquire the ability to pass readily among the humans and cause a pandemic. Scientists are closely monitoring what looks like the birth of a super strain of one of humankind's oldest and most persistent enemies, the influenza virus. This new strain has the potential to kill hundreds of millions given the right conditions. Avian influenza viruses are species specific but do occasionally cross the species barrier to infect humans and other mammals.
Avian influenza $\mathrm{H} 5 \mathrm{~N} 1$ is posing serious concerns to the medical community due to its stunning killing ability, a statistic known as the lethality of the disease. Influenza virus membranes contain two glycoproteins: haemagglutinin and neuraminidases. The influenza A virus particle or virion is $80-120 \mathrm{~nm}$ - It contains eight pieces of segmented negative-sense RNA (13.5 kilobases total), which encode 11 proteins (HA, NA, NP, M1, M2, NS1, NEP, PA, PB1, PB1-F2, PB2).Viral proteins are haemagglutinin and neuraminidase, two large glycoproteins found on the outside of the viral particles (Rupert et al. 2006).

Haemagglutinin mediates cell-surface sialic acid receptor binding to initiate virus infection. After virus replication, neuraminidase removes sialic acid from virus and cellular glycoproteins to facilitate virus release and the spread of infection to new cells1. The distinct antigenic properties of

*Author for correspondence: abhibiotek@gmail.com 
different haemagglutinin and neuraminidase molecules are used to classify influenza type A viruses into subtypes: 16 for haemagglutinin (H1H16) and 9 for neuraminidase (N1-N9) (Von et al. 1993). Numerous combinations of haemagglutinin and neuraminidase subtypes are found in avian species. The $\mathrm{N} 1$ and $\mathrm{N} 2$ neuraminidases of viruses currently circulating in humans belong to two phylogenetically distinct groups. Group- 1 contains $\mathrm{N} 1, \mathrm{~N} 4, \mathrm{~N} 5$ and N8 subtypes whereas group-2 contains N2, N3, N6, N7 and N9.

Neuraminidase has been targeted in structurebased enzyme inhibitor design programmes that have resulted in the production of two drugs, zanamivir (Relenza) (Von Itzstein et al. 1993) and oseltamivir (Tamiflu) (Kim C. U. et al. 1997). Reports of the emergence of drug resistance make the development of new anti-influenza molecules a priority(Aeron C et al. 2006, Jackson et al. 2000).

Structure of H5N1 avian influenza neuraminidase suggests new opportunities for drug design. Present investigation was undertaken to carry out the docking procedure using different ligands from Stemona tuberosa to find out potential inhibitor for $\mathrm{N} 1 \mathrm{Neuraminidase}$ of $\mathrm{H} 5 \mathrm{~N} 1$ avian virus.

Stemonaceae is a small family with four genera, namely, Croomia, Pentastemona, Stemona and Stichoneuron. Stemona is the largest genus with about 32 species, whereas the other genera each have one or two species. They are distributed in Asia, tropical Australia and North America.

Stemona tuberosa is a species registered in the Chinese Pharmacopoeia.The water extracts of the root tuber of this plant are used in Chinese, Japanese and Korean traditional medicines to treat respiratory disorders, e.g., bronchitis, pertussis and tuberculosis, and also as an anthelmintic agent for domestic animals. The roots of Stemona genus (Stemonaceae) have long been prescribed in traditional Chinese medicine as insecticidal and antitussive agents. Extracts from the roots of these plants are used for respiratory disorders, including pulmonary tuberculosis and bronchitis, and externally used against different insect pests (Michael Adams et al. 2005, Li-Gen et al. 2008).

\section{MATERIALS AND METHODS}

NCBI， Genbank，PDB， PROSITE， Pubchem databases were used to get the sequence and structure information. Pre ADMET server was used for AMDET calculation of drug molecules.
AUTODOCK 4 a docking analysis software was used to study interaction of drug molecules with the target. Chem draw tool was used to draw the structures of drug molecules used for analysis. Various compounds present in Stemona tuberosa was tested for activity against $\mathrm{H} 5 \mathrm{~N} 1$ neuraminidase in silico. Eighty one molecules including stilbenoids, bibenzyls and others were selected as probable compounds for lead molecules.

Neuraminidase has been targeted for structure based enzyme inhibitor drug design programmes as a result of which two drugs Zanamivir and oseltamivir has been designed. The X-ray molecular structure of the influenza virus has already been determined (Varghese et al. 1983, Baker et al. 1987). Structure of Neuraminidase was retrieved from Protein Data Bank and used for analysis.

\section{RESULTS AND DISCUSSIONS}

\section{Bioinformatics analysis of neuraminidase sequence \\ Whole genome analysis result}

In the whole genome analysis there was a conserved region in the amino acid region 145156. This range of amino acid was mainly focused to find the docking site in structural analysis. Genome sequence data also shown that reassortment involving all the segments of the influenza virus genome, is a frequent process and might also facilitate major antigenic changes. Natural selection on the HA protein seemed to operate in a punctuated manner, causing distinct but irregular episodes of phenotypic change that were manifested as antigenic 'cluster jumps'.

\section{Validation of compounds from S. tuberosa}

Validation of compounds was carried out using pre ADMET server. The Compounds which cleared toxicity assay and follow lipinski rule were chosen for docking analysis. Out of 81 molecules, 33 molecules cleared validation test.

\section{List of validated compounds}

From Table 1, molecules which are non mutagen are validated compounds which were used for further analysis. Docking of validated ligands with active site of protein was carried out using Auto dock. 
Using Pre ADMER server it was verified that Croomine satisfies Lipinski's Rule of Five and also clears the mutagenecity tests. Molecular weight of Croomine is 307.388800 daltons.
Number of Hydrogen bond acceptors and donors are 4 and 1 respectively. Croomine is negative for TA98, TA100, TA1525 strains of Ames mutagenicity Tests.

Table 1 - List of compounds from Stemona tuberosa which were subjected to validation tests in pre ADMET server.

\begin{tabular}{|c|c|c|}
\hline Number & Compound Name stilbenoids (S1-S25) \& Other compounds & Compound type \\
\hline 1 & $\mathrm{~S} 1$ & Mutagen \\
\hline 2 & S2 & Mutagen \\
\hline 3 & S3 & Non -Mutagen \\
\hline 4 & S4 & Mutagen \\
\hline 5 & S5 & Non -Mutagen \\
\hline 6 & S6 & Non -Mutagen \\
\hline 7 & S7 & Non -Mutagen \\
\hline 8 & S8 & Non -Mutagen \\
\hline 9 & S9 & Non -Mutagen \\
\hline 10 & S10 & Non -Mutagen \\
\hline 11 & S11 & Non -Mutagen \\
\hline 12 & $\mathrm{~S} 12$ & Non -Mutagen \\
\hline 13 & S13 & Mutagen \\
\hline 14 & S14 & Mutagen \\
\hline 15 & S15 & Non-Mutagen \\
\hline 16 & S16 & Mutagen \\
\hline 17 & S17 & Non-Mutagen \\
\hline 18 & S18 & Non -Mutagen \\
\hline 19 & S19 & Mutagen \\
\hline 20 & $\mathrm{~S} 20$ & Mutagen \\
\hline 21 & $\mathrm{~S} 21$ & Mutagen \\
\hline 22 & $\mathrm{~S} 22$ & Mutagen \\
\hline 23 & $\mathrm{~S} 23$ & Mutagen \\
\hline 24 & $\mathrm{~S} 24$ & Mutagen \\
\hline 25 & $\mathrm{~S} 25$ & Mutagen \\
\hline 26 & Stenine & Non-Mutagen \\
\hline 27 & Tuberostemonine & Non -Mutagen \\
\hline 28 & Neotuberostemonine & Non -Mutagen \\
\hline 29 & Croomine & Non -Mutagen \\
\hline 30 & Stemonine & Non-Mutagen \\
\hline 31 & 3,5-dihydroxy-4-methyl bibenzyl & Mutagen \\
\hline 32 & 3,5-dihydroxy-2-methoxy-4-methylbibenzyl & Mutagen \\
\hline 33 & 3 hydroxy-2,5-dimethoxy-2-methyl bibenzyl & Mutagen \\
\hline 34 & Bisdehydrostemonine & Mutagen \\
\hline 35 & Iso Bisdehydrostemonine & Non -Mutagen \\
\hline 36 & Bisdehydrostemonine A & Non -Mutagen \\
\hline 37 & Bisdehydrostemonine B & Non -Mutagen \\
\hline 38 & Bisdehydrostemonine $\mathrm{C}$ & Mutagen \\
\hline 39 & Bisdehydrostemonine D & Mutagen \\
\hline 40 & Bisdehydrostemonine $\mathrm{E}$ & Mutagen \\
\hline 41 & Protostemonine & Mutagen \\
\hline 42 & Stemanthraquinone & Mutagen \\
\hline 43 & Phenanthraquinone & Mutagen \\
\hline 44 & Resveratrol & Mutagen \\
\hline 45 & Pinosylvin A & Mutagen \\
\hline 46 & Pinosylvin B & Mutagen \\
\hline
\end{tabular}




\begin{tabular}{|c|c|c|}
\hline Number & Compound Name stilbenoids (S1-S25) \& Other compounds & Compound type \\
\hline 47 & Pinosylvin $\mathrm{C}$ & Mutagen \\
\hline 48 & Pinosylvin D & Mutagen \\
\hline 49 & Stemofoline & Mutagen \\
\hline 50 & Dihydropinosylvin & Mutagen \\
\hline 51 & Iso Dihydropinosylvin & Mutagen \\
\hline 52 & Stilbostemin A & Mutagen \\
\hline 53 & Stilbostemin B & Non -Mutagen \\
\hline 54 & Stilbostemin C & Mutagen \\
\hline 55 & Stilbostemin D & Non -Mutagen \\
\hline 56 & Stilbostemin E & Mutagen \\
\hline 57 & Stilbostemin F & Non -Mutagen \\
\hline 58 & Stilbostemin G & Non -Mutagen \\
\hline 59 & Stemokerrine & Mutagen \\
\hline 60 & Stemofuran A & Mutagen \\
\hline 61 & Stemofuran B & Mutagen \\
\hline 62 & Stemofuran C & Non-Mutagen \\
\hline 63 & Stemofuran D & Non-Mutagen \\
\hline 64 & Stemofuran E & Mutagen \\
\hline 65 & Stemofuran & Mutagen \\
\hline 66 & Stemanthrene A & Mutagen \\
\hline 67 & Stemanthrene C & Non-Mutagen \\
\hline 68 & Stemanthrene D & Non -Mutagen \\
\hline 69 & Stemanthrene E & Non -Mutagen \\
\hline 70 & Zileuton & Mutagen \\
\hline 71 & Parvistemonine A & Mutagen \\
\hline 72 & Parvistemonine B & Mutagen \\
\hline 73 & 6-hydroxycroomine & Mutagen \\
\hline 74 & Stemocurtisinol & Mutagen \\
\hline 75 & Stemocurtisine & Mutagen \\
\hline 76 & Tuberostemonine $\mathrm{H}$ & Non-Mutagen \\
\hline 77 & Neostemonine & Non -Mutagen \\
\hline 78 & Prostemonine & Non -Mutagen \\
\hline 79 & Maistemonine & Non -Mutagen \\
\hline 80 & Stemonidine & Non-Mutagen \\
\hline 81 & Stemotinine & Non -Mutagen \\
\hline
\end{tabular}

Table 2 - Comparative docking results of ligands having lesser binding energy values among validated compounds in comparison with Zanamivir and Oseltamivir.

\begin{tabular}{lc}
\hline Compound Name & $\boldsymbol{\Delta G}(\mathbf{K c a l} / \mathbf{m o l})$ \\
\hline Croomine & -7.45 \\
Stemonine & -7.41 \\
Tuberostemonie & -7.14 \\
Stilbostemin G & -6.98 \\
Stilbostemin C & -6.93 \\
Zanamivir & -6.60 \\
Oseltamivir & -4.85 \\
\hline
\end{tabular}






Figure 1 - Interaction of croomine with active site of H5N1 Neuraminidase captured using Autodock 4.

\section{CONCLUSION}

The worldwide spread of H5N1 avian influenza virus has raised concerns that this virus might acquire the ability to pass readily among humans, and cause pandemic. Various validated compounds from $S$. tuberosa were used as inhibitor for $\mathrm{N} 1$ neuraminidase of $\mathrm{H} 5 \mathrm{~N} 1$ avian virus. Results showed that some compounds are better than currently used anti-influenza drugs. Hence $S$. tuberosa could be expected as a silver lining for latest threat to mankind, H5N1 Pandemic.

\section{REFERENCES}

Rupert J. Russell, The structure of H5N1 avian influenza neuraminidase suggests new opportunities for drug design, Nature .2006;443(2):45-49.

Von Itzstein M,Rational design of potent sialidasebased inhibitors of influenza virus replication. Nature. 1993; 363(4):418-423.

Kim C. U,Influenza neuraminidase inhibitors possessing a novel hydrophobic interaction in the enzyme active site: design, synthesis, and structural analysis of carbocyclic sialic acid analogues with potent antiinfluenza activity", J. Am. Chem. Soc. 1997; 119(6):681-690.
Aeron C.Hurt, Pina Innello, Neruminidase InhibiotorResistant and -Sensitive Influenza B Viruses Isolated from an Untreated Human Patient,Antimicrobial Agents and Chemotherapy, 2006;50(3):1872-1874.

Jackson H.C.,N.Roberts,Z.M. Wang, and R.Belshe, Management of influenza :use of new antivirals and resistance in perspective, Clin.Drug Investig.2000; 20(7): 447-454.

Varghese, J.N., Laver, W.G \& Colman, P.M,Structure of influenza virus glycoprotein antigen neuraminidase at 2.9 A Resolution, Nature 1983;303(5):35-40.

Baker, A.T, Varghese J.N,LaverW.G, \& Colman, P.M, Three -dimensional structure of neuraminidase of subtype N9 from an avian influenza virus,Proteins 1982; 34(3):111-117.

Michael Adams, Thomas Pacher, Harald Greger and Rudolf Bauer ,Inhibition of Leukotriene Biosynthesis by Stilbenoids from Stemona Species, J. Nat. Prod. 2005;68(9): 83-85.

Li-Gen Lin, Xin-Zhou Yang, Chun-Ping Tang, ChangQiang K,Antibacterial stilbenoids from the roots of Stemona tuberose, Phytochemistry , 2008;69(1) :457463.

Received: January 12, 2010; Revised: July 01, 2011; Accepted: February 19, 2012. 